\title{
Difficulty is critical: Psychological factors in modeling diffusion of green products and practices
}

\author{
Katarzyna Byrka ${ }^{\mathrm{a}}$, Arkadiusz Jędrzejewski ${ }^{\mathrm{b}}$, Katarzyna Sznajd-Weron ${ }^{\mathrm{c}}$, Rafał Weron ${ }^{\mathrm{b}}$ \\ ${ }^{a}$ Institute of Social Psychology, University of Social Sciences and Humanities, Wrocław, Poland \\ ${ }^{b}$ Department of Operations Research, Wroctaw University of Technology, Poland \\ ${ }^{c}$ Department of Theoretical Physics, Wroctaw University of Technology, Poland
}

\begin{abstract}
Despite the very positive - as measured by market surveys - attitude towards eco-innovations and sustainability in general, the actual market penetration of green products and practices generally falls behind the expectations. In this paper we argue that considering difficulty of engagement, as used in the Campbell Paradigm, is of critical importance when modeling diffusion of ecoinnovations. Such a notion of difficulty possesses three desired properties: (i) parsimony - it is represented by a single value, (ii) interpretability - it can be regarded as an estimator of the otherwise complex notion of behavioral cost, and (iii) applicability - it can be easily measured through market surveys. In an extensive simulation and analytical study involving empirically measured difficulty and an agent-based model spanned on different social network structures, we show that innovation adoption may exhibit abrupt changes in market penetration as a result of even small changes in difficulty. The latter may be of particular interest to policy makers who have to make strategic decisions when introducing socially - but not necessarily individually - desired products and practices, like dynamic or green electricity tariffs.
\end{abstract}

Keywords: Green products and practices, Energy policy, Innovation diffusion, Difficulty, Social network, Agent-based model.

\section{Introduction}

In response to climate change, low-carbon policies have been recently enforced worldwide. Effective transition to green energy, however, depends not only on efforts of policy makers, but also on customers' acceptance of novel products or ideas [1-4]. Survey research shows that most customers hold positive attitudes towards eco-innovations. At the same time, adoption rates of solutions such as dynamic electricity tariffs remain unsatisfactorily low [5-7]. The observed discrepancy, also known as the intention-behavior gap, yields the need to identify factors that affect adoption rates. And as Gyamfi et al. [8] argue, social psychology and economic behavior models should be used to overcome these challenges.

Email addresses: kbyrka@swps.edu.pl (Katarzyna Byrka), arekjd@gmail . com (Arkadiusz Jȩdrzejewski), katarzyna.weron@pwr .edu.pl (Katarzyna Sznajd-Weron), rafal . weron@pwr.edu.pl (Rafał Weron) 
A well explored factor that fosters diffusion of green products and practices is conformity to others [9]. Innovations such as dynamic electricity tariffs naturally involve uncertainty triggered by insufficient knowledge. In such situations of uncertainty, opinions and behaviors of friends and neighbors often serve people as a guideline for their own behaviors [10,11]. Accordingly, in empirical research peer effects were found to increase adoption of photovoltaic solar panels [12] or reduce residential energy usage at households [13-15]. Conformity has been also considered in agent-based models (ABM), in particular in the context of adoption of dynamic electricity tariffs [7], green consumption patterns [16], electric vehicles and sustainable transport [17-19] or energy technologies in households [20-22].

A somewhat less studied factor but potentially equally relevant is the cost related to adoption that may effectively hinder this process [23-25]. If external barriers are modeled in the literature at all, they refer to price regulations and financial burden set on customers $[7,16]$. However, turning on one's washing machine after midnight to benefit from lower prices of electricity requires rescheduling of daily chores and vigilance at nighttime. We argue here that adoption of sustainable products and practices bears costs that go well beyond financial expenses and that ignoring this fact may result in unreliable analyzes and forecasts.

The contribution of this paper is threefold. Firstly, we develop a novel approach to agent-based modeling of the adoption of eco-innovations, in which - besides the effect of social influence (i.e. conformity to neighbors or peers) - we introduce external costs defined as difficulty of adoption of a given behavior. Secondly, we demonstrate that difficulty is not an abstract construct but can be measured empirically in a relatively straightforward way. Finally, using survey data from the Netherlands and Poland, we show that the differences in difficulty can be interpreted as costs of different energy-related, sustainable behaviors in cross-country comparisons.

The remainder of the paper is structured as follows. In Section 2 we provide a comprehensive review the most important features of a good ABM for modeling diffusion of green products and practices. Next, in Section 3 we introduce the notion of difficulty of engagement, as used in the Campbell Paradigm, and argue that it possesses three desired properties: (i) parsimony - it is represented by a single value, (ii) interpretability - it can be regarded as an estimator of the otherwise complex notion of behavioral cost, and (iii) applicability - it can be easily measured through market surveys. In Section 4 we show how difficulty can be efficiently implemented in an agent-based model of eco-innovation diffusion. First, in Section 4.1 we briefly describe the Monte Carlo modeling framework, then in Section 4.2 we provide analytical calculations for a complete graph (i.e. a fully connected network of agents). In Section 5 we present and discuss the obtained results. Finally, in Section 6 we wrap up the results and discuss policy implications.

\section{What makes a good eco-innovation diffusion model?}

It is not easy to define what makes a good model for the diffusion of green products and practices. There are many approaches, even within agent-based modeling (ABM) itself [26]. Like for any model design, simplicity and reality (or usefulness) are the key factors. However, as Jager and Mosler [27] nicely put it, the challenge resides in simplifying the often complex theories of social science and the complex reality into simple sets of rules. 
In this Section we discuss the most important - in our opinion - features of a good ABM for modeling diffusion of eco-innovations and comment on how they can be implemented. Before we move on, we would like to emphasize that (i) although focused on diffusion of sustainable products and practices, in some aspects the following discussion reaches far beyond this modeling context and that (ii) all models - no matter how good - are only simplistic representations of reality and should not be used in a mechanical way for the conduct of policy, definitely not without allowing for judgmental adjustment.

\subsection{Binary opinions are realistic (enough)}

One of the pivotal elements to be decided when building an innovation diffusion model is how to describe the agents (also referred to as actors, consumers, customers, individuals or spinsons). The simplest, most natural and probably also the most popular choice is to define an agent by a single dichotomous variable that represents an adopted $(+1)$ or an unadopted $(0)$ state. The term spinson (= 'spin' + 'person'; coined by Nyczka and Sznajd-Weron [28]) reflects exactly this characteristic - the dichotomous nature of the consumers - and has its origins in spin models of statistical physics and humanly features of the agents.

The idea of binary states - which we will also utilize in this paper - is natural from the social point of view, since dichotomous response format with 1 (yes, true, agree) and 0 (no, false, disagree) as response options is one of the most common in social experiments [29]. Interestingly, it can be traced back to Schelling's model of racial segregation or even to the famous Lenz-Ising model of ferromagnetism proposed already in the 1920s [30]. However, agents with more than two states have been also considered in the literature. For instance, Moldovan and Goldenberg [31] defined three states: uninformed, adopters and resisters, while Deffuant et al. [32] introduced a preadoption state in which an interested individual was ready to adopt, but took reflection time to be sure of the decision. Interest in the innovation was defined as a three state variable ('no', 'maybe' or 'yes') and its value was dependent on the global opinion and uncertainty in the society. Janssen and Jager [33] considered so-called consumats, which were characterized by two continuous variables that described their specific individual taste in two dimensions.

\subsection{Social response is not only conformity}

The second pivotal element to be considered is the precise definition of rules that change the state of the agent, i.e. the representation of consumers' decision making processes. In innovation diffusion models we usually assume that the state of an agent is affected by external influence (e.g. advertising, mass media) and internal influence, i.e. interactions between the agents (e.g. word-ofmouth). To model the latter various rules are used [26]. Many of these rules, including epidemic processes and threshold models, rely on imitating the behavior of others $[17,18,34]$. Therefore they describe only one type of social response - conformity. However, conformity is not the only type of social response $[30,35]$.

In this study we consider an agent-based model with two types of social response-conformity and independence. We are fully aware that independence is not the only type of non-conformity and, for instance, anti-conformity could be also taken into account as in [28]. However, including both types of non-conformity makes the model more complicated and, hence, more difficult to 
analyze and interpret. Besides, it is always good practice to start with a simpler approach in order to control the role of all parameters, then expand it if needed.

\subsection{The size of the group matters}

In the so-called q-voter model [36] that we consider here, each individual interacts with a set of $q$ neighbors (i.e. a $q$-lobby) instead of all of its neighbors, like usually in the threshold models $[28,34,37]$. This assumption follows the idea introduced by Galam [38, 39] to model opinion dynamics and is based on the observation that people usually discuss in small groups. The optimal group size for discussions, collaboration, etc., has been the issue of interest for years, mainly from the management of view. For example, it has been shown recently that groups containing 3 to 6 members were significantly more productive and more developmentally advanced than larger groups [40]. In fact, already over four decades ago, Hackman and Vidmar [41] concluded from a cross-sectional study that the optimal team size was 4.6 members.

The size of the group is also very important in the context of social influence. On one hand, the size of the group cannot be too small, i.e. it has to be of a sufficient size to invoke the social pressure. On the other, the size of the group cannot be too large, because people tend to discuss in small groups. It has been observed in a number of social experiments and described by several social theories that for social influence it is not only essential for the majority to be unanimous but also of sufficient size. Increasing the size of the majority will have no additional impact. The threshold value of the group size varies between 3 and 5, depending on the experiment [42].

Moreover, Weiss et al. [43] have recently observed that the infection-spreading models, such as percolation and generalized contagion or epidemic processes, are not consistent with the experimental data. On the contrary, they conclude that approaches utilizing opinion dynamics are able to reproduce the empirical data and are suitable for innovation diffusion modeling.

For the above mentioned reasons, we have decided not to use threshold models, which assume that at the same time all neighbors - no matter how many there are - influence the agent. Looking for a compromise, we have decided to consider here groups of influence that consist of $q=4$ members. We are aware, that one would expect the 'group sizes', represented by parameter $q$, to be sampled from some probability distribution instead of always having the exact same size, but in such a case we would have to assume $a d$ hoc what is the distribution of $q$. Our approach may be interpreted as selecting an optimal or an average group size, which is particularly reasonable if we expect that the variance of the group size distribution is small.

\subsection{Unanimity vs. majority}

As already mentioned, conformity can be modeled in different ways. In the most general approach utilizing threshold models [18, 28, 37], a predefined ratio $r$ of adopted neighbors is needed to influence an agent. Therefore the majority rule corresponds to $r=\frac{1}{2}$, whereas unanimity to $r=1$. Although the majority or supermajority (e.g. $\frac{2}{3}$ or $\frac{3}{4}$ of votes) rules are typically used in voting systems, it has been observed in number of social experiments that a small unanimous group may be more efficient in convincing others than a much larger group with a non-unanimous majority. In the now-classical series of experiments on conformity, Asch [44] found that the presence of a social supporter reduced conformity dramatically - participants of the experiment were far more independent when they were opposed by a seven person majority and had a partner 
sharing the same opinion than when they were opposed by a three-person majority and did not have a partner. Influence of a consistent minority on the responses of a majority has been also reported in [45]. Also recent neurological experiments suggest that unanimous opinions may be critical for normative influence [46]. Moreover, the need for unanimity and the ambiguity of the decision in the absence of a unanimous majority has been considered in a number of social experiments, see [42] for a review. Therefore, it seems that for modeling the diffusion of green products and practices, which involves a high level of uncertainty, the unanimity rule is the most appropriate.

\subsection{Person vs. situation-driven independent behavior}

In real societies agent independence is probably described by a bell-shaped curve, as many other quantitative traits in nature. However, given the complexity and our limited understanding of the human nature and lack of good quality empirical data this is not certain. Independence might instead be better described by a skewed distribution, as wealth, or a multi-modal distribution. In a recent study, Sznajd-Weron et al. [47] have concentrated on two extreme approaches: personspecific and situation-specific. In the person approach there is a fraction $p$ of individuals that are permanently immune and a fraction $(1-p)$ of agents who are susceptible to the social influence. Within such an approach the distribution of independence consists of two peaks - one of height $p$ at $p=1$ and the second of height $(1-p)$ at $p=0$, and can be treated as an approximation of a bi-modal distribution. In the situation approach all agents have the same level of independence $p$, which means that every individual behaves independently with probability $p$ and conforms to group pressure with probability $(1-p)$. Within such an approach the distribution of independence consists of only one peak of height 1 at $p=p$ and can be treated as an approximation of a unimodal bell-shaped curve. Sznajd-Weron et al. [47] have shown that such two extreme scenarios introduced on the micro (i.e. agent) level yield very different results on the macroscopic (i.e. system) level. As there is no consensus on the prevailing type of influence, see [48] for a historical account and a review of the longstanding and vigorous person-situation debate, in this study we confront the results for both approaches - person- and situation-based.

\section{Difficulty of engagement}

Difficulty is considered a key predictor of behavior in social sciences and not surprisingly it is included in the most recognized models of behavior change such as the Theory of Planned Behavior [49] or the Socio-Cognitive Theory [50]. In most of these models estimates of behavioral difficulty rely on people's perceptions. That is, difficulty is considered to be subjective and persondependent [51]. Obviously, difficulty of behaviors - such as using renewable energy sources or dynamic electricity tariffs - may be perceived differently by different individuals. Nevertheless, for a number of reasons a more objective, person-independent difficulty seems more relevant for energy policy. Research shows that for some behaviors the objective difficulty is not perfectly related to its subjective perceptions made by people [52]. In other words, sometimes people fail to recognize how challenging the barriers that they have to overcome really are. Moreover, perceived difficulty is not stable over time and as many human perceptions it may depend on respondents' mood or specific circumstances [53]. Because policy makers require reliable indicators of behavioral change, a person-independent difficulty appears to be a more useful concept. 


\subsection{Campbell Paradigm and the Rasch model}

Kaiser et al. [54] have recently proposed a model of behavior change with person-independent difficulty and called it the Campbell Paradigm. Their model, grounded on Campbell's [55] original idea from the 1960s, treats the likelihood of individual behavior as a function of a person's attitude and of the difficulty of engaging in this behavior. This difficulty stems from all sorts of external barriers, both physical and figurative. The more demanding these barriers are, the more favorable attitude towards a general goal, such as environment protection, a person needs to have to overcome them. Kaiser et al. [54] have proposed that the relation between difficulty of behaviors, attitude and behaviors can be measured using a one-parameter logistic Rasch model [56]. Technically, in this model, behavioral difficulty is not estimated on the basis of people's evaluations and opinions (i.e. their subjective perception of 'difficulty') but using the proportion of persons that engage in a given behavior. The probability of engagement in a certain behavior, $\pi_{i s} \in[0,1]$, is modeled as a logistic function of the difference between person $\theta_{s}$ and item (or behavior) $\delta_{i}$ parameters:

$$
\pi_{i s}=\frac{\exp \left(\theta_{s}-\delta_{i}\right)}{\exp \left(\theta_{s}-\delta_{i}\right)+1},
$$

where $i=1, \ldots, N_{i}, s=1, \ldots, N_{s}, N_{i}$ is the number of items (or behaviors) and $N_{s}$ is the number of subjects (or individuals) under study. Recall that an item can be a self-reported or an overt behavior or any other question on a scale [56]. Note, that in the Campbell Paradigm the 'person' parameters pertain to the level of attitude (e.g. pro-environmental attitude) and the 'item' parameters to the realization costs of a particular behavior or - simply put - to the difficulty of engagement. The higher a person's attitude relative to the difficulty of a behavior, the higher the probability that a person will engage in a given behavior.

Difficulty of specific behaviors can be measured on the basis of self-reported behaviors in relatively large samples. Polytomous-format questions can be further converted to a dichotomous format to simplify the analysis and increase precision of estimates [57]. Then, given a certain pattern of responses to all items by all individuals, i.e. an $N_{i} \times N_{s}$ matrix of $\pi_{i s}$ 's with 1 's representing endorsement of a behavior and 0's representing non-endorsement, each person's $\hat{\theta}_{s}$ and each item's $\hat{\delta}_{i}$ can be estimated using maximum likelihood [58].

\subsection{An empirical study: Cross-country comparison}

Questionnaire data with self-reported behaviors can be used to compare external constrains and barriers that amount to difficulty of specific sustainable behaviors. Barriers such as climate, infrastructure or wealthiness of citizens and legislation all affect difficulty and are relatively similar at the country level, especially in the European context [59]. Therefore, for illustrative purposes these administrative units can be used to demonstrate the effects of external conditions affecting people's performance of particular behaviors. For example, one may expect that refraining from using air-conditioning will be far more difficult in Sicily than in Ireland.

Using a similar approach as in [60], we present a comparison of a set of self-reported proecological behaviors of Dutch and Polish citizens from general adult populations. The two countries not only differ in terms of affluence of their citizens, but also with respect to weather conditions, legislative solutions and the infrastructure. We expect, therefore, differences in the difficulty 
of some behaviors. Samples from Eindhoven (the Netherlands) and from Wrocław (Poland) were taken for the cross-country comparison. Dutch data was collected in 2004 by sending questionnaires via regular mail to the citizens of Eindhoven, randomly selected from the municipality register. Out of 4445 approached persons 1746 sent back completed questionnaires (response rate of $39.7 \%$ ), either spontaneously or after one reminder. After exclusion of persons below the age of 18, 1703 individuals were included in the analysis. The mean age of participants was 47.5 years and there were 847 female $(49.7 \%)$ and 856 male $(50.3 \%)$ respondents. Polish data was collected in 2012 by personal approaching of 1272 persons at their households. The addresses of the participants were randomly selected from the municipality register as well. Of these 1272 individuals, 463 persons returned completed questionnaires (response rate of $36.4 \%$ ). The mean age of participants was 41.6 years, $277(59.8 \%)$ were females and $186(40.2 \%)$ were males.

The 50 self-reported ecological behaviors (or items) came from the well-established General Ecological Behavior (GEB) scale [57] and could be a priori assigned to one of the domains, such as energy use, transportation, consumerism, recycling, and vicarious behaviors. Of the 50 behaviors, 18 had a dichotomous yes (1) or no (0) response format. Responses meant either endorsement or non-endorsement of a certain ecological behavior. An exemplary item would be: I am a member of a carpool. However, behaviors that are performed recurrently could not be responded to in such a way. Therefore, 32 items had a polytomous format and were responded to on a five-level response scale from never (1) to always (5), for instance, the item I ride a bicycle or take public transportation to work or school. The answers to polytomous-format questions were converted before the analysis to a dichotomous format by treating the responses never, seldom and occasionally as non-endorsement (0) and often and always as endorsement (1). Such a recoding procedure is a confirmed standard for this scale and was shown to increase precision of estimates [57].

Typically, in a Rasch-type analysis, one expects that difficulties of items generalize across persons. In the assessment of the accuracy of this generalization (or the 'item fit') we relied on the mean square (MS) statistics weighted by the item variance [61]. For example, the averaged MS of 1.20 corresponds to $20 \%$ excess of variation in empirical data compared to the model's prediction. The analysis revealed that all 50 items in both samples showed reasonable fit and fell within the accepted limit of $\mathrm{MS}<1.20$ [58].

Formula (1) translates a person's attitude $\theta_{s}$ minus an item's difficulty $\delta_{i}$ to probability $\pi_{i s}$ of engagement of this person in this behavior. As discussed in Section 3.1, in practical applications formula (1) is fed with a pattern of responses to all items by all individuals, $\pi_{i s} \in\{0,1\}$, to yield estimates of $\delta_{i}$ and $\theta_{s}$ for all $i=1, \ldots, N_{i}$ and $s=1, \ldots, N_{s}$. Now, if we introduce an attitude of an average citizen in a given country and compute it as the sample average, i.e. $\bar{\theta}=\frac{1}{N_{s}} \sum_{s=1}^{N_{s}} \theta_{s}$, the resulting probabilities:

$$
\pi_{i}=\frac{\exp \left(\bar{\theta}-\delta_{i}\right)}{\exp \left(\bar{\theta}-\delta_{i}\right)+1}
$$

are probabilities that an average citizen will engage in item $i$. They can serve as information about external barriers that can hinder an average citizen in a given country to perform this behavior (of difficulty $\delta_{i}$ ). From formula (2) it follows that probability of engagement $\pi_{i}$ decreases with difficulty $\delta_{i}$, i.e. the higher the difficulty the lower the probability of performance, which agrees with the intuitive concept of difficulty. Using the above formula we estimated engagement likelihoods 

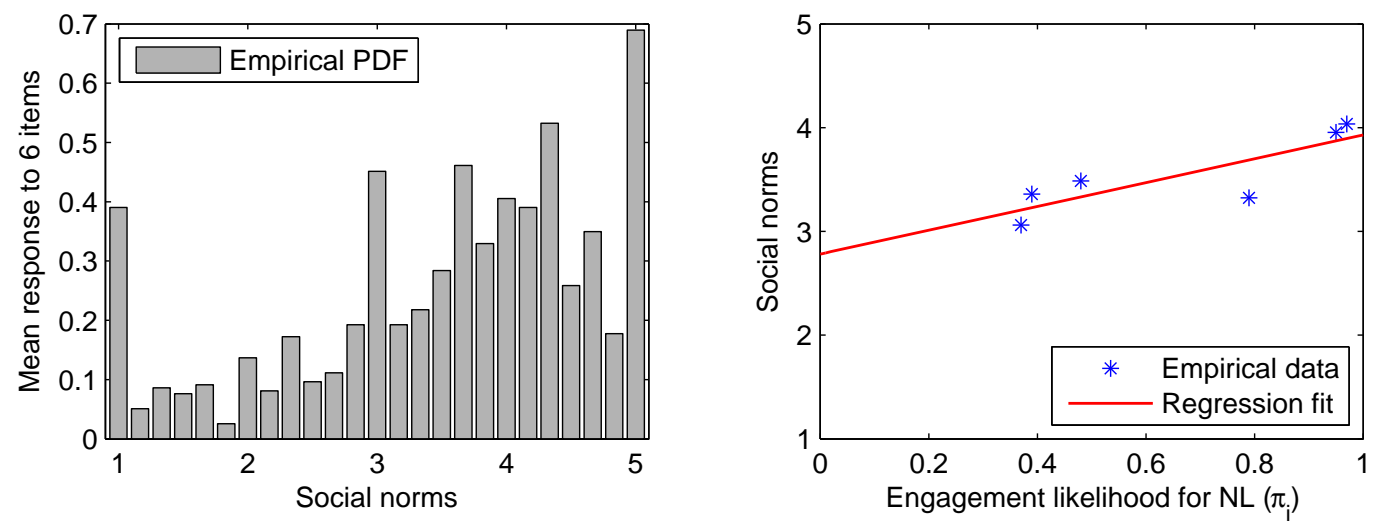

Figure 1: Left panel: Empirical probability density function (PDF; i.e. a normalized histogram) of the mean response in the Dutch sample to six items indicated by a star in Table 1, on a 1 to 5 scale. Right panel: Social norms as a function of engagement likelihoods, $\pi_{i}$, and a linear regression fit. See Section 3.3 for a discussion.

for each behavior for a citizen with an average level of pro-environmental attitude.

The results, summarized in Table 1, show that certain behaviors appear to be more demanding in Poland than in the Netherlands. Other behaviors, vice versa, are more likely, and appear easier, to be performed in Poland. The observed differences in difficulty of behaviors in the two countries can be attributed to external conditions that are specific for Poland and the Netherlands [59]. For example, the Poles generally refrain from using clothes driers (probability of refraining $\pi_{49}$ is as high as 0.96 ), while the Dutch are more likely to use such appliances $\left(\pi_{49}\right.$ is only 0.39$)$. One of the explanations can be the humid, Atlantic climate that makes drying clothes without a tumble drier impossible in the Netherlands. Additionally, the salary of the Poles is on average lower than of the Dutch (despite the decade separating both samples) and many cannot afford buying the expensive device. At the same time, using renewable energy sources seems far easier for the Dutch $\left(\pi_{7}=0.41\right)$ than for the Poles $\left(\pi_{7}=0.15\right)$. This difference can be explained by a better accessibility of alternative sources of energy and more frequent awareness raising campaigns in the Netherlands compared to Poland. Of course, finding specific reasons for observed differences in difficulties requires additional research. Nonetheless, it seems plausible to accept that external, person-independent conditions translate into objective and person-independent difficulty of behaviors. Moreover, this difficulty should not be ignored in the process of fostering novel behaviors, in which adoption of innovative solutions (such as renewable energy sources) still remains rather low.

\subsection{Social norms, difficulty of engagement and agent-based modeling}

In the previously mentioned questionnaire distributed to the Dutch sample we additionally measured social norms using a standard set of questions within the Theory of Planned Behavior. The participants responded to six questions composed of the phrase Most of the people that are important to me think that I should ... and of one of the six behaviors related to environment conservation, i.e. the six behaviors marked by an asterisk $(*)$ in Table 1 . The participants indicated the likelihood of each situation on a five-option format scale, i.e. choosing from unlikely (1) to 
Table 1: Engagement (or performance) likelihoods $\pi_{i}$ for 50 pro-ecological behaviors in Poland (PL) and in the Netherlands (NL) estimated with the one-parameter Rasch-model, see formula (2), for a person with an average proecological attitude. Behaviors (also called items) in bold are energy-related. Asterisks $\left(^{*}\right)$ indicate the six behaviors used in the social-norms measure in the Dutch sample, see Fig. 1 and Section 3.3.

\begin{tabular}{|c|c|c|c|}
\hline \multirow[t]{2}{*}{ No. } & \multirow[t]{2}{*}{ Pro-ecological behaviors (items) } & \multicolumn{2}{|c|}{$\begin{array}{c}\text { Engagement } \\
\text { likelihoods }\left(\pi_{i}\right)\end{array}$} \\
\hline & & $\mathbf{P L}$ & NL \\
\hline 1. & I am a member of an environmental organization & 0.02 & 0.16 \\
\hline 2. & I contribute financially to environmental organizations & 0.05 & 0.11 \\
\hline 3. & I bought solar panels to produce energy & 0.06 & 0.02 \\
\hline 4. & At red traffic lights, I switch off the engine & 0.07 & 0.02 \\
\hline 5. & In winter, I do not leave the windows open for long periods of time & 0.09 & 0.33 \\
\hline 6. & I requested an estimate on having solar power installed & 0.12 & 0.02 \\
\hline 7. & I use renewable energy sources & 0.15 & 0.41 \\
\hline 8. & I buy unbleached and uncolored toilet paper & 0.18 & 0.34 \\
\hline 9. & I have pointed out unecological behavior to someone & 0.18 & 0.12 \\
\hline 10 . & I refrain from using fabric softener with my laundry & 0.20 & 0.46 \\
\hline 11. & I talk with friends about problems related to the environment & 0.20 & 0.08 \\
\hline 12. & I buy meat and produce with eco-labels. & 0.20 & 0.07 \\
\hline 13. & I refrain from owning a car & 0.21 & 0.06 \\
\hline 14. & I boycott companies with an unecological background & 0.27 & 0.10 \\
\hline 15 . & I buy milk in returnable bottles & 0.29 & 0.05 \\
\hline 16. & I read about environmental issues & 0.30 & 0.13 \\
\hline 17. & I buy furniture made from domestic woods & 0.36 & 0.90 \\
\hline 18. & I drive on freeways at speeds under $100 \mathrm{kph}(=62.5 \mathrm{mph})$ & 0.38 & 0.27 \\
\hline 19. & I take public transportation to where I want to start my hikes & 0.43 & 0.48 \\
\hline 20. & In winter, I keep the heat low so that I have to wear a sweater & 0.42 & 0.87 \\
\hline 21. & I use a chemical air freshener in my bathroom & 0.47 & 0.53 \\
\hline 22. & I refrain from using an oven cleaning spray to clean my oven & 0.49 & 0.87 \\
\hline 23. & *I collect and recycle used paper & 0.50 & 0.97 \\
\hline 24. & I have already looked into the pros and cons of having a private source of power & 0.51 & 0.10 \\
\hline 25. & ${ }^{*}$ I switch off the engine while waiting in front of a railroad crossing or in a traffic jam & 0.51 & 0.34 \\
\hline 26. & I refrain form driving my car in or into the city & 0.53 & 0.43 \\
\hline 27. & If I am offered a plastic bag in a store, I never take it & 0.54 & 0.12 \\
\hline 28. & In nearby areas (ca. $30 \mathrm{~km}$ or 20 miles), I use public transportation or ride a bike & 0.54 & 0.43 \\
\hline 29. & *I buy products in refillable packages & 0.49 & 0.36 \\
\hline 30 . & In winter, I turn down the heat when I leave my apartment for more than 4 hours & 0.59 & 0.86 \\
\hline 31. & ${ }^{*}$ I ride a bicycle or take public transportation to work or school & 0.62 & 0.56 \\
\hline 32. & I refrain from killing insects with a chemical insecticide & 0.64 & 0.73 \\
\hline 33. & I am a member of a carpool & 0.65 & 0.12 \\
\hline 34. & I refrain from buying beverages in cans & 0.67 & 0.72 \\
\hline 35 . & I bring empty bottles to a recycling bin & 0.70 & 0.96 \\
\hline 36. & I own a fuel-efficient automobile (less than 7 litters per $100 \mathbf{~ k m}$ ) & 0.71 & 0.53 \\
\hline 37. & ${ }^{*}$ I wash dirty clothes without pre-washing & 0.72 & 0.78 \\
\hline 38. & I put dead batteries in the recycle bit & 0.75 & 0.90 \\
\hline 39. & I refrain from buying convenience foods & 0.77 & 0.74 \\
\hline 40 . & For longer journeys (more than 6 hours), I never take an airplane & 0.78 & 0.53 \\
\hline 41. & After meals, I never dispose of leftovers in the toilet & 0.78 & 0.85 \\
\hline 42. & In hotels, I reuse the towels & 0.80 & 0.64 \\
\hline 43. & I drive in such a way as to keep my fuel consumption as low as possible & 0.80 & 0.76 \\
\hline 44. & I rather take a shower than a bath & 0.82 & 0.90 \\
\hline 45 . & I buy seasonal produce & 0.85 & 0.84 \\
\hline 46. & ${ }^{*}$ I wait until I have a full load before doing my laundry & 0.88 & 0.95 \\
\hline 47. & I own energy efficient household devices & 0.89 & 0.78 \\
\hline 48. & I reuse my shopping bags & 0.96 & 0.99 \\
\hline 49. & I refrain from using a clothes dryer & 0.96 & 0.39 \\
\hline 50. & After a picnic, I leave the place as clean as it was originally & 0.98 & 1.00 \\
\hline
\end{tabular}


likely (5). Since social norms are perceived as social pressure from important others to engage or not to engage in a given behavior, higher agreement with such norms can be interpreted as conformity to peer pressure [49]. On the other hand, lower agreement with such norms can be interpreted as independence, a concept that will be used in Section 4.

Having information about person-independent difficulty and responses to social norm questions in reference to the six behaviors we can compare whether perceived pressure from others is dependent or not on the objectively measured difficulty. As can be seen in Figure 1, the higher the estimated likelihood of performance $\pi_{i}$ (i.e. the less difficult the behavior), the higher is the perceived pressure from the others to engage in a given behavior. The results suggest that people are probably aware of the constraints set on very difficult behaviors and subjective conviction of the social pressure with respect to these behaviors is relatively low. It seems that perceived social norms, and probably conformity to opinions from others as well, will be different for different levels of behavioral difficulty. As social pressure and conformity lie at the heart of many agent-based models of innovation diffusion, our results speak for the necessity of introducing difficulty to such models.

To date, difficulty understood as external constraints has been treated in agent-based models exclusively in reference to financial costs. For instance, Bravo et al. [16] developed an ABM in which the rise of prices of organic food or of green energy resulted in a lower consumption of these goods. Here we argue that adoption of some behaviors goes beyond financial costs. For example, using dynamic electricity tariffs requires reorganization of daily routines, rescheduling of activities of all household inhabitants. A more comprehensive approach to difficulty, as presented in the Campbell Paradigm, takes into account all inconveniences set on behaviors. To the best of our knowledge, so far no agent-based model has treated difficulty in such a way.

\section{An agent-based model of eco-innovation diffusion}

We consider a network of $N$ nodes, each occupied by exactly one spinson, i.e. an agent characterized by a binary opinion about the innovation. Opinion $S_{i}= \pm 1$ of spinson $i$ is a dynamic variable and can change in time. Furthermore, each spinson is described by a trait - level of independence $p_{i} \in[0,1]$, which is a static variable and does not change in time. In the situation setup, see Section 2.5, all agents are homogenous and behave independently with probability $p_{i}=p$ and conform with probability $(1-p)$. In the person setup there are two groups of agents: $N_{1}=p N$ independent spinsons with $p_{i}=1$ and $N_{2}=(1-p) N$ conformists with $p_{i}=0$. We consider two types of social response:

- Independence - with probability $p_{i}$ spinson $i$ acts independently, i.e. takes an opinion regardless of the social pressure. The only factor that influences spinson's opinion is the difficulty of engagement, understood as in the Campbell Paradigm. The more difficult the adoption of the innovation is, i.e. the higher the probability of non-engagement $f=(1-\pi) \equiv\left(1-\pi_{i}\right)$, the more likely the spinson is to flip against it. With probability $f$ the spinson takes a state against adoption and with probability $(1-f)$ the spinson adopts.

- Conformity - with probability $\left(1-p_{i}\right)$ the spinson is susceptible to the social influence. If all agents in a randomly selected $q$-panel of neighbors share the same opinion the spinson 

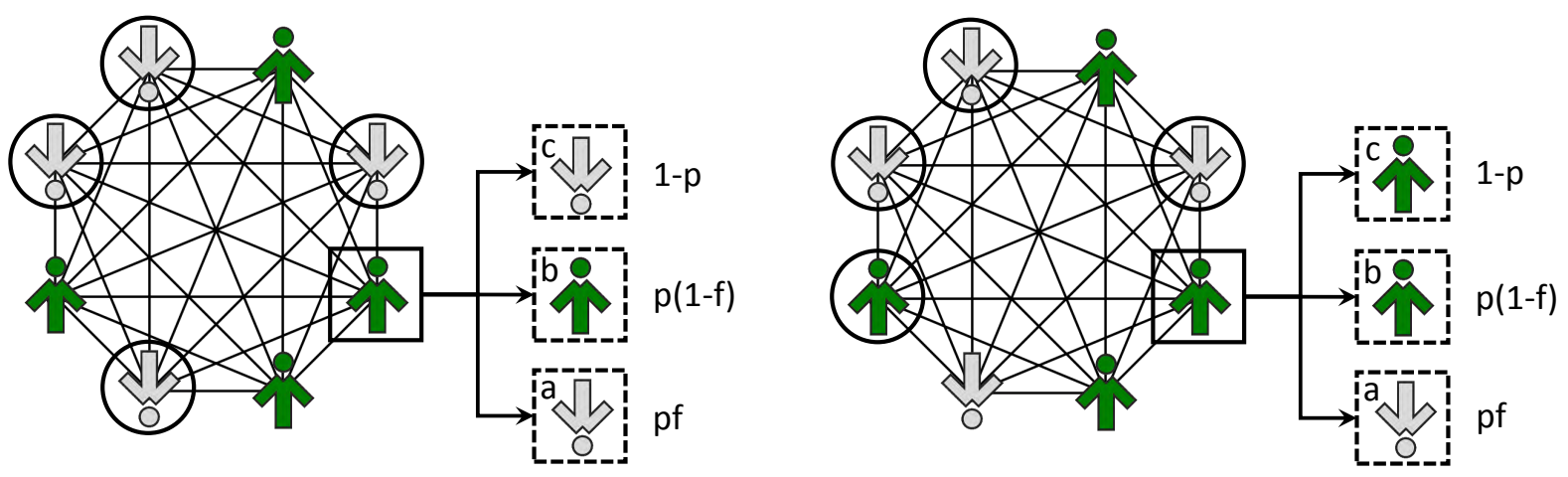

Figure 2: Visualization of the model defined by steps 1-4 in Section 4. First, we randomly choose a spinson (step 1) indicated by a solid square. If it is independent (step 2; $p$ is spinson's independence), then in step 3 with probability of non-engagement $f$ the spinson takes a state against adoption (a; both panels) and with probability $(1-f)$ the spinson adopts ( $\mathrm{b}$; both panels). Otherwise the spinson is susceptible to the social influence (step 4). We randomly choose $q=4$ neighbors - indicated by solid circles. If they all share the same opinion then the spinson conforms to the group (c; left panel); if there is no unanimous majority the spinson stays in the previous state (c; right panel).

takes the same state.

Recall that higher agreement with social norms can be interpreted as conformity to peer pressure, while lower agreement with such norms - as independence [10]. Hence, the approach described in Section 3.3 can potentially be used to estimate the level of independence in the society. The calibration of the probability of non-engagement is easier - it can be simply computed as one minus the probability of engagement, see Section 3.2 and Table 1.

In general we can investigate the model on an arbitrary graph but in this paper we will limit considerations to only two types of structures:

- Complete graph - in which every node is connected with every other node. This type of structure is suitable for describing small social groups or cliques in arbitrary social structures. It also allows for analytical treatment.

- Small world network - described by the Watts-Strogatz [62] algorithm. Within such an algorithm we start with a one-dimensional regular lattice of size $N$ with periodic boundary conditions in which each node is connected to its $K$ neighbors. Then with probability $\beta$ we replace each edge by a randomly chosen one. This kind of structure is interesting for several reasons. For $\beta \in[0.01,0.1]$ it exhibits properties observed in real-life social networks, such as short path length and high clustering. Note that in this study we present results only for small world networks with $\beta=0.05$, however, we have checked other $\beta$ 's in this range and the results were qualitatively the same. Moreover, small world networks allow to tune the structure from the regular one $(\beta=0)$ to a completely random $(\beta=1)$. Finally, for $\beta=0$ and $K=N-1$ the network reduces to a complete graph, which is also of interest to us. 


\subsection{Monte Carlo simulations}

To study the model we conduct Monte Carlo simulations for systems of size $N=10^{4}$ spinsons. The obtained results are averaged over $10^{4}$ samples (i.e. system evolution trajectories) after reaching the stationary state. In the simulations the time is measured in Monte Carlo steps (MCS). A single MCS consists of $N$ elementary sequential updates (see Fig. 2):

1. Randomly choose a single spinson $(\rightarrow i)$.

2. With probability $p_{i}$ the spinson acts independently (go to 3 ), otherwise the spinson is susceptible to the social influence (go to 4 ).

3. With probability of engagement $(1-f)=\pi$ the spinson adopts or stays adopted, i.e. set $S_{i}=1$, and with probability $f$ the spinson unadopts or stays unadopted due to the high difficulty of engagement, i.e. set $S_{i}=-1$. Go to 1 .

4. Randomly choose $q$ neighbors of the spinson. If they all share the same opinion then the spinson takes the same state, i.e. it conforms to the group. If there is no unanimous majority the spinson does not change its state. Go to 1.

In the initial state all agents are unadopted, i.e. all spinsons are down: $S_{i}=-1$ for $i=1, \ldots, N$. This corresponds to the situation prior to introducing the innovation (e.g. a new type of solar panels, a new electricity tariff) when none of the agents is adopted. What triggers adoption is independence, as some spinsons start to flip. Then social influence from a unanimous group of $q=4$ neighbors may influence a spinson. Note that on a complete graph all spinsons are neighbors, hence, the group of influence is chosen randomly from all $N-1$ spinsons. However, on a small world network the $q$ neighbors can be chosen randomly only from the $n_{i} \geq 4$ spinsons directly connected (i.e. sharing a link) with spinson $i$. In our simulations we set $K=8$. In the rare cases that after rewiring the network not all nodes have at least 4 neighbors, we build a new network to ensure that all nodes have the required number of neighbors. Note that Janssen and Jager [33] used an alternative approach to assure that all agents had at least 4 neighbors - they started with a regular network with $K=4$ and randomly connected each other possible pair of agents with probability $1 \%$. However, by doing so they increased the average degree of their small-world network.

\subsection{Analytical calculations for a complete graph}

In this Section we perform analytical calculations to validate our MC simulations. For an arbitrary network it is usually impossible to compute how the number of adopted spinsons changes in time and what is the stationary state. However, in the case of a complete graph this task turns out to be very simple $[30,47]$. On such a network structure all spinsons are connected with each other (they are all neighbors) and the system is completely homogeneous in the sense that the local ratio of adopted spinsons is statistically equal to the global ratio. Therefore we can write down the equation that describes the evolution of the system.

In the person approach there are two groups of agents: $N_{1}=p N$ independent spinsons and $N_{2}=(1-p) N$ conformists. For each of these two groups we can write the equation describing the time evolution of the number of adopted spinsons. Using the notation introduced in [47], let $N_{1}^{\uparrow}(t)$ and $N_{1}^{\downarrow}(t)$ denote respectively the number of adopted and unadopted independent spinsons at time 
$t$. Therefore, the ratio of adopted independent spinsons at time $t$ is equal to $c_{1}(t)=N_{1}^{\uparrow}(t) / N$. Similarly, let $N_{2}^{\uparrow}(t)$ and $N_{2}^{\downarrow}(t)$ denote respectively the number of adopted and unadopted conformists at time $t$ and the ratio of adopted conformists at time $t$ is equal to $c_{2}(t)=N_{1}^{\uparrow}(t) / N$. Carefully analyzing steps 1-4 of the simulation algorithm described in Section 4, we can derive the probabilities of all events that increase or decrease the ratio of adopted in an elementary time step. Finally we obtain:

$$
\begin{aligned}
c_{1}^{\prime} & =c_{1}+\frac{1}{N}\left[\left(1-c_{1}\right)(1-f)-c_{1} f\right], \\
c_{2}^{\prime} & =c_{2}+\frac{1}{N}\left[\left(1-c_{2}\right) c^{4}(t)-c_{2}\left(1-c^{4}\right)\right], \\
c^{\prime} & =c_{1}+c_{2},
\end{aligned}
$$

where, for brevity of notation, $c_{1,2} \equiv c_{1,2}(t)$ and $c_{1,2}^{\prime} \equiv c_{1,2}(t+1)$.

In the situation approach all agents are homogenous and behave independently with probability $p$ and conform with probability $(1-p)$. Therefore:

$$
c^{\prime}=c+\frac{1}{N}\left[(1-c)\left\{p(1-f)+(1-p) c^{4}\right\}-c\left\{p f+(1-p)\left(1-c^{4}\right)\right\}\right] .
$$

From formulas (3) and (4) we can obtain the stationary value of the ratio of adopted spinsons, i.e. $c=c(\infty)$, and it agrees very well with Monte Carlo results, as can be seen in Figs. 3-4 where analytical results for a complete graph are plotted as solid lines.

\section{Results}

We investigate our model using Monte Carlo simulations described in Section 4.1 and the analytical approach discussed in Section 4.2. In the former case we simulate a system of size $N=10^{4}$ spinsons and average the results over $10^{4}$ samples (i.e. system evolution trajectories). Starting from the initial, completely unadopted state the system evolves according to the algorithm described in Section 4. Eventually the system reaches a stationary state in which the ratio of adopted fluctuates around some average value $c=c(\infty)$. These values are plotted as symbols in Figs. 3-4 and as solid lines in Fig. 5.

Let us first focus on the dependence between the ratio of adopted $c$ and independence $p$ for a fixed value of non-engagement $f$, see Fig. 3. Note the reverse scale on the horizontal axes (for consistency with the 3D plots in Fig. 5). The results are presented for two social network structures - a complete graph (left panels) and a small world network (right panels) - and two opinion dynamics - 'person' (top panels) and 'situation' (bottom panels). For difficult behaviors $(f>0.5)$, the ratio of adopted increases with independence $p$ but it never reaches high values. This means that for a high level of non-engagement the adoption fails, although a small fraction of spinsons can adopt. Very much alike a pro-environmental behavior that is difficult yet adopted by a small part of the society. Moreover, for difficult behaviors $(f>0.5)$ the results are almost the same for both network structures and there are only minor differences between the results for the person and situation approaches. In other words, changes in the network structure cannot help the 

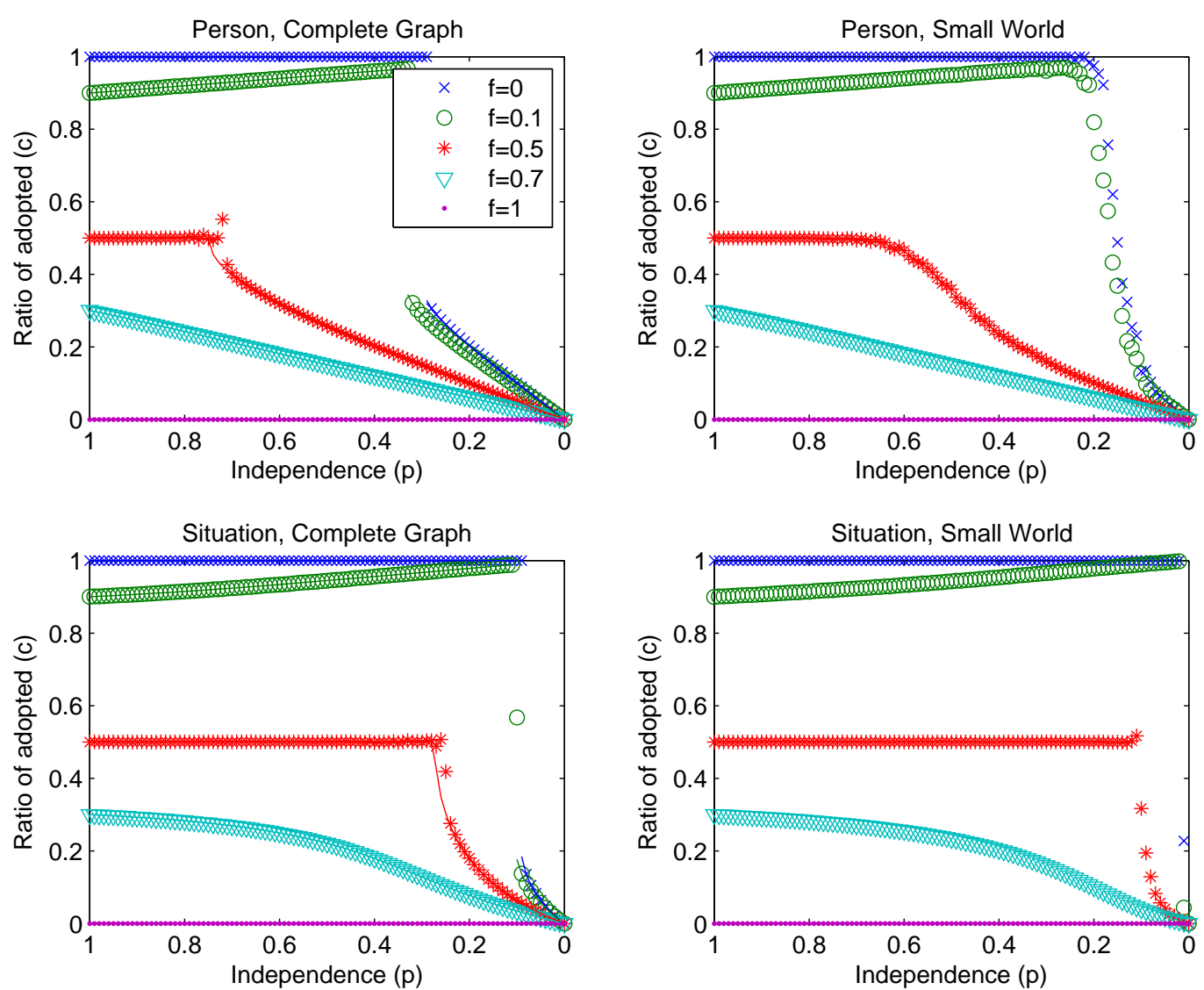

Figure 3: The stationary value of the ratio of adopted $c$ as a function of independence $p$ for several values of nonengagement $f$. Results are presented for two social network structures - a complete graph (left panels) and a small world network (right panels) - and two opinion dynamics - 'person' (top panels) and 'situation' (bottom panels). Symbols indicate Monte Carlo simulation results, whereas solid lines analytical results (only for a complete graph). Note the reverse scale on the horizontal axes.

diffusion and the only possibility to increase the ratio of adopted is to decrease non-engagement below the threshold value of $f=0.5$.

Below this value, i.e. for less difficult innovations $(f \leq 0.5)$, a very interesting behavior is observed and differences between network structures and two types of opinion dynamics (person vs. situation) are revealed. Generally, as a result of competition between conformity, which is an ordering force, and independence, which introduces noise and disorders the system, a strongly non-linear behavior can be observed. Below a certain critical value of independence, $p_{c}$, ratio of adopted $c$ increases systematically with independence $p$, then at $p=p_{c}$ the ratio of adopted jumps to a high value, close to $c=1$. For larger values of independence, $p>p_{c}$, the ratio of adopted slowly decreases but still remains high. This slight decrease for $p>p_{c}$ is caused by too large independence, which destroys the consensus (order). Interestingly, the person-oriented model on a small-world network shows a somewhat different behavior - no jump is observed. Nevertheless, the general behavior is similar in all cases.

Even more interesting results are obtained if we analyze the dependence between the stationary 

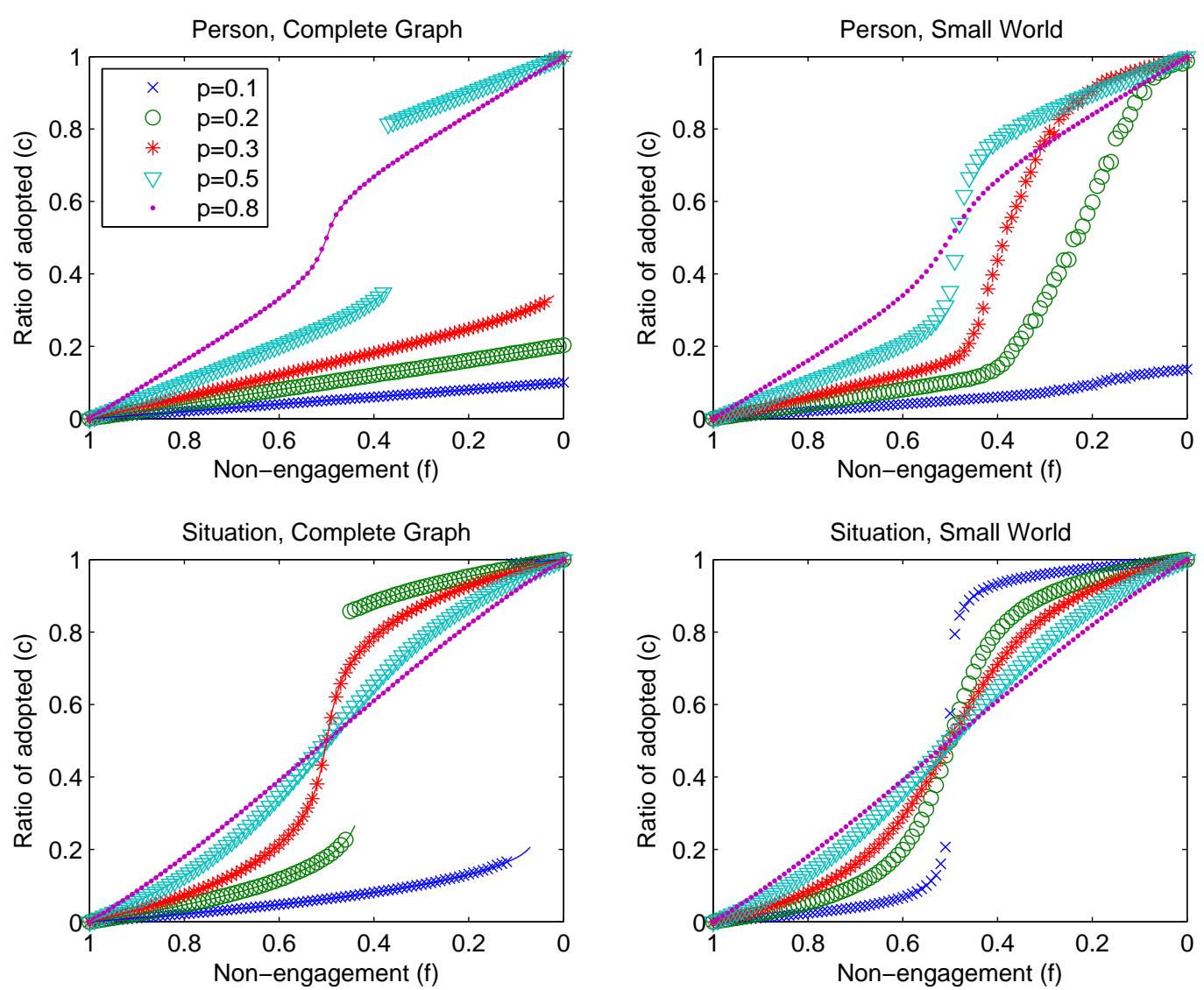

Figure 4: The stationary value of the ratio of adopted $c$ as a function of non-engagement $f$ for several values of independence $p$. Results are presented for two social network structures - a complete graph (left panels) and a small world network (right panels) - and two opinion dynamics - 'person' (top panels) and 'situation' (bottom panels). Symbols indicate Monte Carlo simulation results, whereas solid lines analytical results (only for a complete graph). Note the reverse scale on the horizontal axes.

ratio of adopted $c$ and non-engagement $f$ for a fixed value of independence $p$, see Fig. 4. Note the reverse scale on the horizontal axes (for consistency with the 3D plots in Fig. 5). Generally, for very high values of independence, which is unlikely from the social point of view, dependence between the ratio of adopted and non-engagement is almost or even completely linear. For smaller values of independence it becomes strongly non-linear and in the case of a complete graph, even discontinuous. Interestingly, discontinuity is not present on the small-world network, which means that all processes run more smoothly in this case. However, strong non-linearity is still present.

Up till now we have considered the ratio of adopted as a function of only one parameter independence $p$ (with a fixed value of non-engagement) or non-engagement $f$ (with a fixed value of independence). The three dimensional (3D) plots in Fig. 5 display the ratio of adopted, $c$, as a function of both $p$ and $f$. Although analyzing 3D graphs may be tricky, it is instructive if we want to compare different models. In general, the results in all four cases are quite similar - except that the person approach on a small-world network does not display a discontinuity (top left panel). However, there are quantitative differences between the approaches. For example, in the situation 


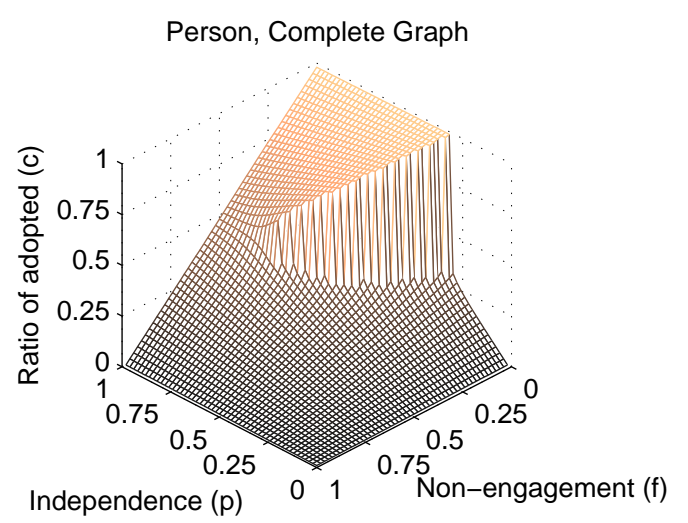

Situation, Complete Graph

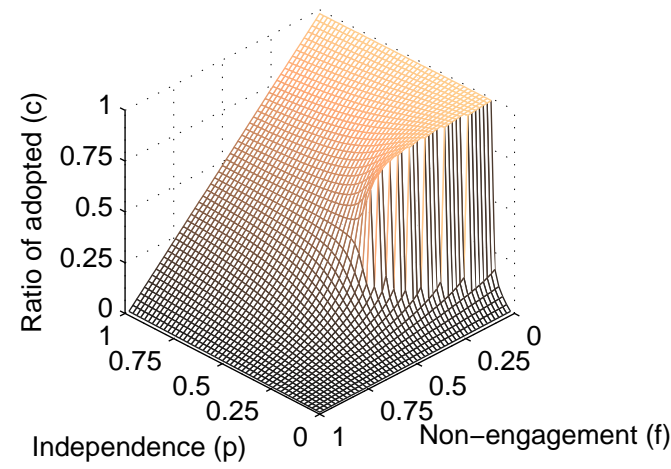

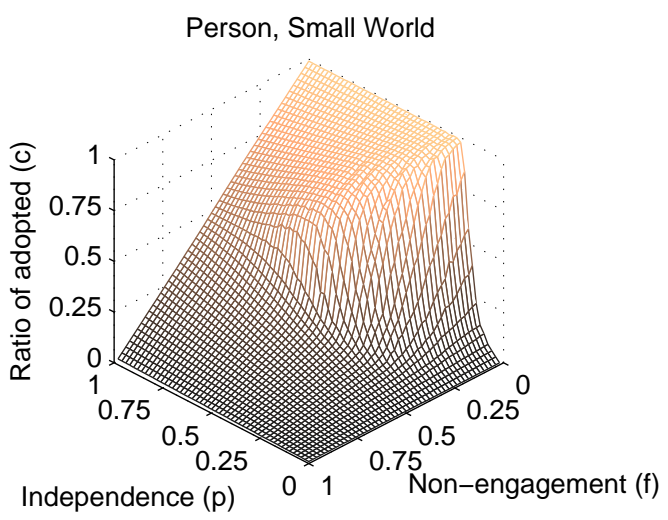

Situation, Small World

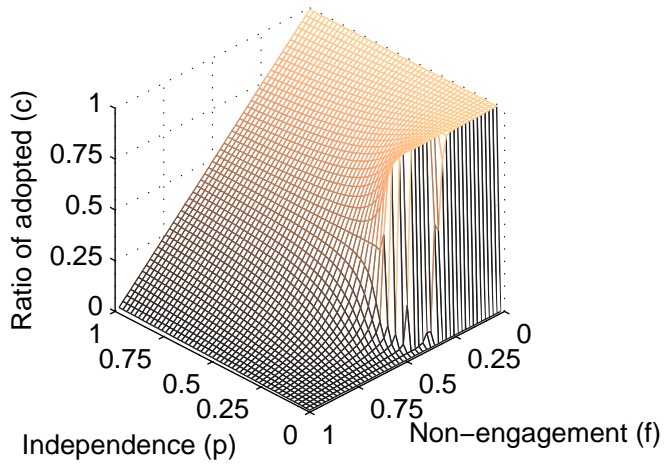

Figure 5: The stationary value of the ratio of adopted $c$ as a function of independence $p$ and non-engagement $f$. Like in Figures 3-4, results are presented for two social network structures - a complete graph (left panels) and a small world network (right panels) - and two opinion dynamics - 'person' (top panels) and 'situation' (bottom panels). These are Monte Carlo simulation results; analytical results (only for a complete graph) are indistinguishable from the $\mathrm{MC}$ results at this scale.

approach, the adoption is possible for lower values of independence (for a small-world network). This means that heterogeneous social networks support the adoption. Nevertheless, the general results are similar in all cases: there is a strong non-linearity associated with $f$. This means that a significant change in the system (e.g. a switch from an unadopted to an adopted society) is possible even as a result of a small change of the difficulty of engagement. From this point of view difficulty is indeed critical.

\section{Conclusions}

In this paper we have proposed and investigated a simple agent-based model, that takes into account two types of social response (conformity and independence), as well as behavioral difficulty that may significantly hamper diffusion of innovation. Interestingly, we have shown that dependence between the ratio of adopted $c$ and non-engagement $f$ is quite complex - varying from linear, through strongly non-linear (s-shaped) to rapid discontinuous dependence. This means that the concept of difficulty is indeed critical for diffusion of innovation. Even a slight change of 

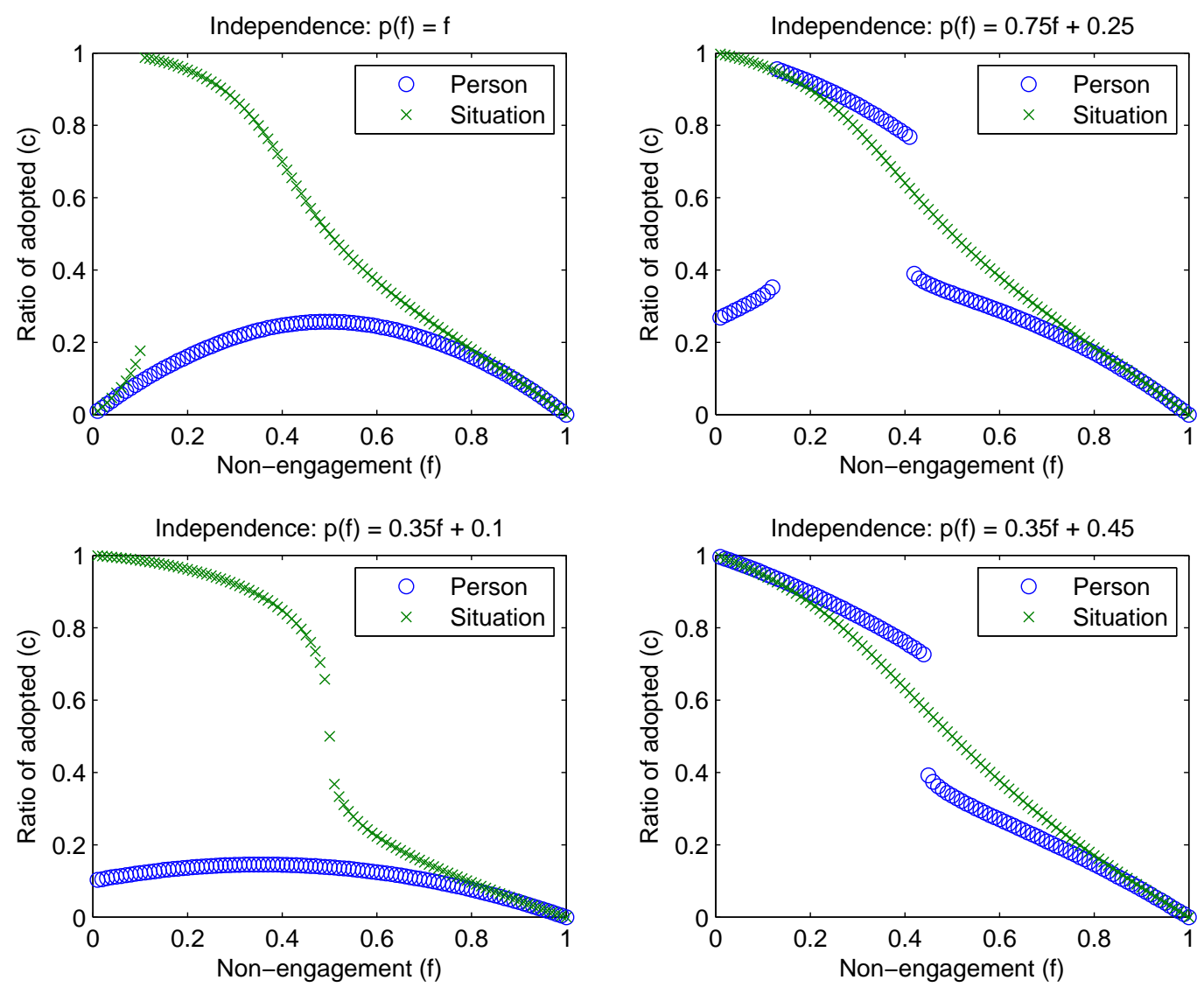

Figure 6: The stationary values of the ratio of adopted $c$ on a complete graph as a function of non-engagement $f$ for two opinion dynamics - 'person' and 'situation' - and four hypothetical scenarios of the functional relationship between $p$ and $f$. These are analytical results for a complete graph. Note the extreme differences in $c$ (as a function of $f$ ) for relatively similar functional forms of $p$.

difficulty may lead to dramatic changes in the success rate of the diffusion of innovation.

Up till now we have treated two parameters of the model - independence $p$ and non-engagement $f$ - as independent variables and we have been investigating systematically the whole parameter space. However, empirical results presented in Section 3.3 suggest that in reality independence may depend on the difficulty of engagement. Preliminary empirical studies (see the right panel in Fig. 1) indicate that this relationship may be linear, i.e. $p(f)=a f+b$. Perhaps this is not a universal form and in general it may be different for different societies. However, even if the relationship is linear, the exact values of coefficients $a$ and $b$ may depend on the studied group. As shown in Fig. 6, there may be extreme differences in the ratio of adopted $c$ (as a function of $f$ ) even for relatively similar functional forms of $p$ ! Therefore, in our opinion it is crucial to accurately estimate the level of independence in the society as a function of non-engagement: $p=p(f)$.

Empirical studies such as ours can help in optimizing the strategy of introducing an innovation. It can provide insight whether a small change in difficulty will result in a success or, on the contrary, even a significant change will not help in the diffusion and it makes no sense to invest more money and time on this particular innovation. We are aware that all results in this study have been 
obtained within a particular agent-based model and therefore may be not completely universal. However, in accordance with good practice, we have compared four versions of the model and all obtained results indicate that difficulty is indeed critical for innovation diffusion.

Considering both conformity and difficulty when modeling diffusion of eco-innovations may provide a plausible explanation as to why adoption rates of generally accepted solutions are lower than expected. It seems that external, not necessarily financial, barriers may prevent customers from adopting green products and practices, even if they are willing and convinced by others to use the sustainable option. We may conclude that in the case of green products and practices, like dynamic electricity tariffs, diffusion of innovation will not proceed spontaneously. Rather, it will have to be additionally fostered by elimination or reduction of costs and barriers that go beyond financial benefits of the customers.

\section{Acknowledgments}

We thank the Technical University of Eindhoven, and particularly Cees Midden and Florian Kaiser, for providing us with the Dutch survey data. We acknowledge financial support of the Foundation for Polish Science under grant no. BIS/2011-3/1 (to KB) and the National Science Center (NCN, Poland) under grant no. NCN 2013/11/HS4/B/01061 (to AJ, KSW and RW).

\section{Bibliography}

[1] O. Edenhofer, R. P. Madruga, and Y. Sokona, eds., Renewable Energy Sources and Climate Change Mitigation. IPCC Special Report, Cambridge University Press, New York, N.Y., 2012.

[2] T. J. Foxon and P. J. Pearson, "Towards improved policy processes for promoting innovation in renewable electricity technologies in the UK," Energy Policy, vol. 35, pp. 1539-1550, 2007.

[3] M. Yazdanpanah, N. Komendantova, and R. S. Ardestani, "Governance of energy transition in Iran: Investigating public acceptance and willingness to use renewable energy sources through socio-psychological model," Renewable and Sustainable Energy Reviews, vol. 45, pp. 565-573, 2015.

[4] N. Zografakis, E. Sifaki, M. Pagalou, G. Nikitaki, V. Psarakis, and K. P. Tsagarakis, "Assessment of public acceptance and willingness to pay for renewable energy sources in Crete," Renewable and Sustainable Energy Reviews, vol. 14, pp. 1088-1095, 2010.

[5] A. Faruqui and J. Palmer, "The discovery of price responsiveness - a survey of experiments involving dynamic pricing of electricity," EDI Quarterly, vol. 4, pp. 15-18, 2012.

[6] Z. Hu, J. ho Kim, J. Wang, and J. Byrne, "Review of dynamic pricing programs in the U.S. and Europe: Status quo and policy recommendations," Renewable and Sustainable Energy Reviews, vol. 42, pp. 743-751, 2015.

[7] A. Kowalska-Pyzalska, K. Maciejowska, K. Suszczyński, K. Sznajd-Weron, and R. Weron, "Turning green: Agent-based modeling of the adoption of dynamic electricity tariffs," Energy Policy, vol. 71, pp. 164-174, 2014.

[8] S. Gyamfi, S. Krumdieck, and T. Urmee, "Residential peak electricity demand response - highlights of some behavioural issues," Renewable and Sustainable Energy Reviews, vol. 25, pp. 71-77, 2013.

[9] H. P. Young, "The dynamics of social innovation," PNAS, vol. 108, pp. 21285-21291, 2011.

[10] R. B. Cialdini and M. R. Trost, Social influence: Social norms, conformity and compliance. McGraw-Hill, 1998.

[11] J. M. Nolan, P. W. Schultz, R. B. Cialdini, N. J. Goldstein, and V. Griskevicius, "Normative social influence is underdetected," Personality and Social Psychology Bulletin, vol. 34, pp. 913-923, 2008.

[12] B. Bollinger and K. Gillingham, "Peer effects in the diffusion of solar photovoltaic panels," Marketing Science, vol. 31, pp. 900-912, 2012.

[13] I. Ayres, S. Raseman, and A. Shih, "Evidence from two large field experiments that peer comparison feedback can reduce residential energy usage," The Journal of Law, Economics, and Organization, vol. 29, pp. 992-1022, 2014. 
[14] E. R. Frederiks, K. Stenner, and E. V. Hobman, "Household energy use: Applying behavioural economics to understand consumer decision-making and behaviour," Renewable and Sustainable Energy Reviews, vol. 41, pp. 1385-1394, 2015.

[15] P. W. Schultz, J. M. Nolan, R. B. Cialdini, N. J. Goldstein, and V. Griskevicius, "The constructive, destructive, and reconstructive power of social norms," Psychological Science, vol. 18, pp. 429-434, 2007.

[16] G. Bravo, E. Vallino, A. K. Cerutti, and M. B. Pairotti, "Alternative scenarios of green consumption in Italy: An empirically grounded model," Environmental Modelling $\mathcal{G}$ Software, vol. 47, pp. 225-234, 2013.

[17] M. J. Eppstein, D. K. Grover, J. S. Marshall, and D. M. Rizzo, "An agent-based model to study market penetration of plug-in hybrid electric vehicles," Energy Policy, vol. 39, pp. 3789-3802, 2011.

[18] D. McCoy and S. Lyons, "Consumer preferences and the influence of networks in electric vehicle diffusion: An agent-based microsimulation in Ireland," Energy Research $\mathcal{F}$ Social Science, vol. 3, pp. 89-101, 2014.

[19] D. Natalini and G. Bravo, "Encouraging sustainable transport choices in American households: Results from an empirically grounded agent-based model," Sustainability, vol. 6, pp. 50-69, 2013.

[20] C. S. Bale, N. J. McCullen, T. J. Foxon, A. M. Rucklidge, and W. F. Gale, "Harnessing social networks for promoting adoption of energy technologies in the domestic sector," Energy Policy, vol. 63, pp. 833-844, 2013.

[21] J. Palmer, G. Sorda, and R. Madlener, "Modeling the diffusion of residential photovoltaic systems in Italy: An agent-based simulation," Technological Forecasting and Social Change, vol. 99, pp. 106-131, 2015.

[22] V. Rai and S. A. Robinson, "Agent-based modeling of energy technology adoption: Empirical integration of social, behavioral, economic, and environmental factors," Environmental Modelling $\mathcal{E}$ Software, vol. 70, pp. 163177, 2015.

[23] T. J. Foxon, R. Gross, A. Chase, J. Howes, A. Arnall, and D. Anderson, "UK innovation systems for new and renewable energy technologies: drivers, barriers and systems failures," Energy Policy, vol. 33, pp. 2123-2137, 2005.

[24] F. G. Kaiser, C. Midden, and R. Cervinka, "Evidence for a data-based environmental policy: Induction of a behavior-based decision support system," Applied Psychology, vol. 57, pp. 151-172, 2008.

[25] B. Wejnert, "Integrating models of diffusion of innovations: A conceptual framework," Annual Review of Sociology, vol. 28, pp. 297-326, 2002.

[26] E. Kiesling, M. Güntherand, C. Stummer, and L. M. Wakolbinger, "Agent-based simulation of innovation diffusion: A review," Central European Journal of Operations Research, vol. 20, pp. 183-230, 2012.

[27] W. Jager and H. J. Mosler, "Simulating human behavior for understanding and managing environmental resource use," Journal of Social Issues, vol. 63, pp. 97-116, 2007.

[28] P. Nyczka and K. Sznajd-Weron, "Anticonformity or independence? - Insights from statistical physics," Journal of Statistical Physics, vol. 151, pp. 174-202, 2013.

[29] R. W. Robins, R. C. Fraley, and R. F. Krueger, eds., Handbook of research methods in personality psychology. Guilford Press, New York, 2007.

[30] P. Przybyła, K. Sznajd-Weron, and R. Weron, "Diffusion of innovation within an agent-based model: Spinsons, independence and advertising," Advances in Complex Systems, vol. 17, p. 1450004, 2014.

[31] S. Moldovan and J. Goldenberg, "Cellular automata modeling of resistance to innovations: Effects and solutions," Technological Forecasting E Social Change, vol. 71, pp. 425-442, 2004.

[32] G. Deffuant, S. Huet, and F. Amblard, "An individual-based model of innovation diffusion mixing social value and individual benefit," American Journal of Sociology, vol. 110, pp. 1041-1069, 2005.

[33] M. A. Janssen and W. Jager, "Fashions, habits and changing preferences: simulation of psychological factors affecting market dynamics," Journal of Economic Psychology, vol. 22, pp. 745-772, 2001.

[34] P. Singh, S. Sreenivasan, B. K. Szymanski, and G. Korniss, "Threshold-limited spreading in social networks with multiple initiators," Scientific Reports, vol. 3, p. 2330, 2013.

[35] P. R. Nail, S. I. D. Domenico, and G. MacDonald, "Proposal of a double diamond model of social response," Review of General Psychology, vol. 17, pp. 1-19, 2013.

[36] C. Castellano, M. A. Muoz, and R. Pastor-Satorras, "Nonlinear q-voter model," Physical Review E, vol. 80, p. 041129, 2009.

[37] M. Granovetter, “Threshold models of collective behavior,” American Journal of Sociology, vol. 83, pp. 14201443, 1978. 
[38] S. Galam, "Majority rule, hierarchical structures and democratic totalitarianism: A statistical approach," Journal of Mathematical Psychology, vol. 30, pp. 426-434, 1986.

[39] S. Galam, Sociophysics: A Physicist's Modeling of Psycho-political Phenomena. Springer, 2012.

[40] S. A. Wheelan, "Group size, group development, and group productivity," Small Group Research, vol. 40, pp. 247-262, 2009.

[41] J. R. Hackman and N. Vidmar, "Effects of size and task type on group performance and member reactions," Sociometry, vol. 33, pp. 37-54, 1970.

[42] R. Bond, “Group size and conformity," Group Processes E Intergroup Relations, vol. 8, pp. 331-354, 2005.

[43] C. H. Weiss, J. Poncela-Casasnovas, J. I. Glaser, A. R. Pah, S. D. Persell, D. W. Baker, R. G. Wunderink, and L. A. N. Amaral, "Adoption of a high-impact innovation in a homogeneous population," Physical Review X, vol. 4, p. 041008, 2014.

[44] S. E. Asch, "Opinions and social pressure," Scientific American, vol. 193, pp. 31-35, 1955.

[45] S. Moscovici, E. Lage, and M. Naffrechoux, "Influence of a consistent minority on the responses of a majority in a color perception task," Sociometry, vol. 32, pp. 365-380, 1969.

[46] D. K. Campbell-Meiklejohn, D. R. Bach, A. Roepstorff, R. J. Dolan, and D. D. Frith, "How the opinion of others affects our valuation of objects," Current Biology, vol. 20, pp. 1165-1170, 2010.

[47] K. Sznajd-Weron, J. Szwabiński, and R. Weron, "Is the person-situation debate important for agent-based modeling and vice-versa?," PLoS ONE, vol. 9, p. e112203, 2014.

[48] M. B. Donnellan, R. E. Lucas, and W. Fleeson, "Introduction to personality and assessment at age 40: Reflections on the legacy of the person-situation debate and the future of person-situation integration," Journal of Research in Personality, vol. 43, no. 2, pp. 117-119, 2009.

[49] I. Ajzen, "The theory of planned behavior," Organizational Behavior and Human Decision Process, vol. 50, pp. 179-211, 1991.

[50] A. Bandura, Social Foundations of Thought and Action: A social cognitive theory. Prentice Hall, Englewood Cliffs, N.J., 1986.

[51] K. Byrka, Attitude-behavior consistency: Campbell's paradigm in environmental and health domains. $\mathrm{PhD}$ thesis, Technische Universiteit Eindhoven, 2009.

[52] I. Ajzen and M. Fishbein, "The influence of attitudes on behavior," in The Handbook of Attitudes (D. Albarracin, B. Johnson, and M. Zanna, eds.), pp. 173-221, Erlbaum, Mahwah, N.J., 2005.

[53] J. S. Lerner and D. Keltner, "Fear, anger, and risk," Journal of Personality and Social Psychology, vol. 81, pp. 146-159, 2001.

[54] F. G. Kaiser, K. Byrka, and T. Hartig, "Reviving Campbell's paradigm for attitude research,” Personality and Social Psychology Review, vol. 14, pp. 351-367, 2010.

[55] D. T. Campbell, "Social attitudes and other acquired behavioral dispositions," in Psychology: A Study of a Science (S. Koch, ed.), vol. 6, pp. 94-172, McGraw-Hill, New York, N.Y., 1963.

[56] S. E. Embretson and S. P. Reise, Item response theory for psychologists. Psychology Press, 2013.

[57] F. G. Kaiser and M. Wilson, "Goal-directed conservation behavior: The specific composition of a general performance," Personality and Individual Differences, vol. 36, pp. 1531-1544, 2004.

[58] T. G. Bond and C. M. Fox, Applying the Rasch model: Fundamental measurement in the human sciences. Erlbaum, Mahwah, NJ, 2001.

[59] F. G. Kaiser and A. Biel, "Assessing general ecological behavior: A cross-cultural comparison between switzerland and sweden," European Journal of Psychological Assessment, vol. 16, pp. 44-52, 2000.

[60] H. Scheuthle, V. Carabias-Hütter, and F. G. Kaiser, "The motivational and instantaneous behavior effects of contexts: Steps toward a theory of goal-directed behavior," Journal of Applied Social Psychology, vol. 35, pp. 2076-2093, 2005.

[61] B. D. Wright and G. N. Masters, Rating scale analysis: Rasch measurement. MESA, Chicago, 1982.

[62] D. J. Watts and S. H. Strogatz, "Collective dynamics of 'small-world' networks," Nature, vol. 393, pp. 440-442, 1998. 